\title{
Nur ein charakteristischer MRT-Befund?
}

Fragestellung: Dieser Artikel fasst die neuesten Ergebnisse zur Bildgebung bezüglich Nachweis, Ätiologie, Prävalenz und Pathophysiologie der kortikalen superfiziellen Siderose mit Schwerpunkt auf der zerebralen Amyloidangiopathie zusammen.

Hintergrund: Unter einer superfiziellen Siderose versteht man lineare Hämosiderinablagerungen im Subarachnoidalraum, den Leptomeningen und in den oberflächlichen Schichten des zerebralen/zerebellären Kortex sowie des Rückenmarks. Sie lassen sich am besten mittels hämsensitiver MRT-Sequenzen nachweisen. Anhand der zugrunde liegenden Pathologie und klinischen Präsentation lassen sich zwei Formen unterscheiden. In diesem Artikel wird speziell auf die supratentorielle kortikale superfizielle Siderose eingegangen,

Charidimou A, Linn J, Vernooij MW et al. Cortical superficial siderosis: detection and clinical significance in cerebral amyloid angiopathy and related conditions. Brain 2015; 138: 2126-39 die die Sulci der Konvexität der Großhirnhemisphären betrifft.

Patienten und Methodik: Es wurden in Pubmed alle relevanten, in Englisch verfassten
Artikel im Zeitraum von 1990 bis Februar 2015 zu den Suchbegriffen „zerebrale Amyloidangiopathie, CAA“, „,kortikale superfizielle Siderose, cSS“ und „kortikale/ fokale Subarachnoidalblutung, cSAH“ ermittelt und berücksichtigt.

Ergebnisse: Obwohl die cSS verschiedene Ursachen haben kann, gilt sie als eines der charakteristischen MRT-Zeichen der CAA, einer häufigen Erkrankung der kleinen Gefäße beim älteren Menschen. Die CAA führt zu intrazerebralen Blutungen und ist mit einer demenziellen Entwicklung assoziiert. Bei Patienten mit einer CAA verursacht die cSS eine typische klinische Symptomatik mit transienten fokalen neurologischen Episoden (TFNE). Erste Untersuchungen weisen darauf hin, dass die cSS ein Warnzeichen für eine im Verlauf entstehende CAA-assoziierte Parenchymblutung ist, was einen möglichen Einfluss auf die gerinnungshemmende Therapie haben kann.

Schlussfolgerungen: Die cSS scheint eine wichtige Rolle bei hämorrhagischen Erkrankungen der kleinen Gefäße in verschiedenen Populationen zu spielen, vor allem bei der CAA, aber auch bei Demenzpatienten und gesunden älteren Menschen.

\section{- Kommentar von Nina Lummel, München}

\section{Hämsensitive MRT-Sequenzen großzügig einsetzen}

In diesem Review-Artikel wird gut strukturiert der aktuelle Stand der Bildgebung und klinischen Bedeutung der kortikalen superfiziellen Siderose (cSS) dargestellt. Insbesondere beim älteren Menschen sollte bei kernspintomografischem Nachweis einer cSS oder einer fokalen kortikalen Subarachnoidalblutung differenzialdiagnostisch in erster Linie an eine CAA gedacht werden. Bei Patienten mit einer CAA verursacht die cSS eine typische klinische Symptomatik mit transienten fokalen neurologischen Episoden (TFNE). Diese werden zum Teil als TIAs oder epileptische Anfälle fehlgedeutet. Zudem kommt die cSS häufiger bei Patienten mit einer Demenz vor. Daher ist das Bild in vielen neurologischen Feldern wie neurovaskulären Erkrankungen, Demenzen und als Differenzialdiagnose von Epilepsien von Bedeutung. Wichtig ist hierbei der großzügige Einsatz hämsensitiver MRT-Sequenzen, um die cSS zu identifizieren. Von besonderem Interesse für den klinischen Alltag ist das Thema der gerinnungshemmenden Therapien bei Patienten mit CAA und cSS. Leider fehlen hierzu evidenzbasierte Daten. Man weiß allerdings, dass das Reblutungsrisiko für Patienten mit vorangegangener CAA-assoziierter intrazerebraler Blutung (ohne Gerinnungshemmung) mit $10 \%$ pro Jahr hoch ist [1]. Und dieses Risiko steigt bei Einnahme gerinnungshemmender Medikamente [1] oder Nachweis einer cSS [2]. Die Autoren empfehlen bei Patienten mit einer wahrscheinlichen CAA (im MRT multiple Mikroblutungen und Lappenblutungen) ein individuelles Abwägen von Nutzen und Risiko bezüglich der Gabe von Antikoagulanzien oder Plätt- chenhemmern, insbesondere wenn eine klare Indikation für die gerinnungshemmende Therapie besteht, die das Risiko einer intrazerebralen Blutung ausgleicht oder überwiegt. Auf jeden Fall scheint eine suffiziente Blutdruckeinstellung bei $\mathrm{Pa}$ tienten mit CAA und cSS sinnvoll, da dies das Risiko für eine intrazerebrale Blutung senkt [3]. Insgesamt ist die derzeitige Datenlage zur cSS noch nicht ausreichend und weitere prospektive Studien sollten folgen. Beispielsweise basiert die Annahme der cSS als Risikofaktor für eine zukünftige intrazerebrale Blutung auf einer einzigen, multizentrischen Studie bei CAA-Patienten.
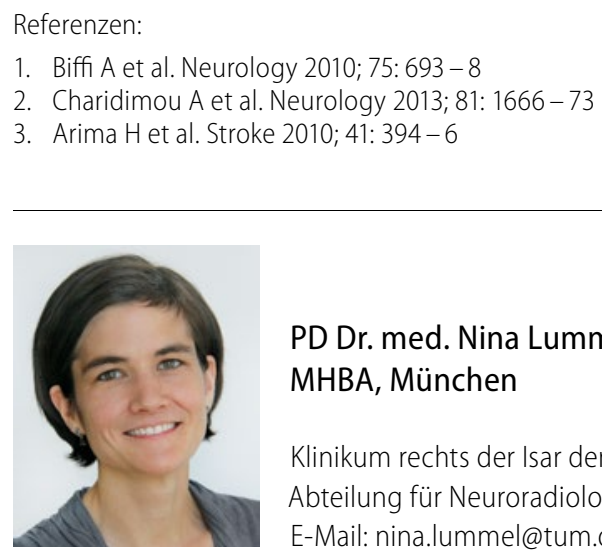

PD Dr. med. Nina Lummel, MHBA, München

Klinikum rechts der Isar der TU München, Abteilung für Neuroradiologie E-Mail: nina.lummel@tum.de 